\title{
Protection of the Inventor Outside the Patent System
}

\section{Dean C. Dunlavey*}

The owner of an invention has resort to two alternative sources of law when seeking to preserve for himself the profit from his invention; he can look either to the common law (sometimes codified, with possible modifications, by state statute) or to the federal patent statute. ${ }^{1}$ The common law imposes no obligation upon the inventor to disclose his invention to others; he is free to keep his secret entirely to himself. ${ }^{2}$ The inventor also has the right, recognized by the common law, to make, use and sell his invention. ${ }^{3}$ This right to practice the invention is of limited value, however, unless it can be kept exclusive. ${ }^{4}$ Hence, it is exclusivity which the inventor primarily seeks from the law.

The aim of the patent system is to encourage inventors to disclose their inventions to the public so that society may benefit from them. ${ }^{5} \mathrm{~A}$ patent grant necessitates a complete disclosure although no use need be made of the invention by the patentee. ${ }^{6}$ In return for such disclosure the government adds to the inventor's common law right to make, use and sell the invention by conferring upon him the right to exclude all others from practicing the invention for a period of seventeen years. ${ }^{7}$ For those inventions

* IL.B. University of California, Berkeley, June, 1955; Faculty Fellow, Harvard Law School, 1955-1956.

166 STAT. 792-817 (1952), 35. U.S.C. \$\$ 1-293 (1952); Becher v. Contoure Laboratories, 279 U.S. 388 (1928) ; Metallizing Engineer Co. v. Kenyon Bearing \& A.P. Co., 153 F.2d 516

(2d Cir. 1946), cert. denied, 328 U.S. 840 (1946); Nye Tool \& Machine Works v. Crown Die \& Tool Co., 276 Fed. 376 (7th Cir. 1921), rev'd on other grounds, 261 U.S. 24 (1922). See also Herrman, Protection of Unpatentable Articles of Manufacture, 21 J. PaT. OrF. Soc'y 959 (1939) (proposing a middle ground between patents and the common law).

2 U.S. v. Dubilier Condenser Corp., 289 U.S. 178 (1932); United States v. Bell Telephone Company, 167 U.S. 224 (1896) ; Bates v. Coe, 98 U.S. 31 (1878) ; Chemical Foundation v. General Aniline Works, 99 F.2d 276 (3rd Cir. 1938), cert. denied, 305 U.S. 654 (1938).

3 Bauer v. ODonnell, 229 U.S. 1 (1912); Six Wheel Corporation v. Sterling Motor Truck Co., 50 F.2d 568 (9th Cir. 1931); Summerhays v. Scheu, 10 Cal.App.2d 574, 52 P.2d 512 (1935).

4 The right of exclusion does not exist at common law. Gaylor et al. v. Wilder, 51 U.S. (10 How.) 477 (1850) ; M. J. Lewis Products Co. v. Lewis, 57 F.2d 886 (E.D. Pa. 1931) ; Tower Mfg. Co., Inc. v. Monsanto Chemical Works, 20 F.2d 386 (S.D.N.Y. 1927).

6 United States v. Bell Telephone Company, 167 U.S. 224 (1896); Kendall et al. v. Wmsor, 62 U.S. (21 How.) 322 (1858).

6 Paper Bag Patent Case, 210 U.S. 405 (1907). See note 12 infra.

766 STAT. 804 (1952), 35 U.S.C. \$ 154 (1952); Crown Co. v. Nye Tool Works, 261 U.S. 24 (1922); Bauer v. O'Donnell, 229 U.S. 1 (1912); Paper Bag Patent Case, 210 U.S. 405 (1907); Bloomer v. McQuewan et al., 55 U.S. (14 How.) 539 (1852); Six Wheel Corporation v. Sterling 
whose exploitation is incompatible with secrecy, the patent system offers the only feasible protection for the inventor.

\section{Common law protection of the inventor's secret}

Exclusivity is afforded by the common law in a much different manner. ${ }^{\circ}$ At common law the public is free to make, use and sell anything of which it has knowledge. ${ }^{10}$ Accordingly, the inventor's right of exclusivity depends solely upon successful exercise of his right to keep the invention a secret, thereby making it impossible for others to duplicate it. ${ }^{11}$ To this end, the common law will protect him against wrongful acquisition and usage by others of knowledge of his invention; ${ }^{12}$ the extent of such protection is a

Motor Truck Co., 50 F.2d 568 (9th Cir. 1931); Tower Mfg. Co., Inc. v. Monsanto Chemical Works, 20 F.2d 386 (S.D.N.Y. 1927).

The patentee's right of exclusion begins with the patent issue. Woodbridge v. United States, 263 U.S. 50 (1923); Gayler et al. v. Wilder, 51 U.S. (10 How.) 477 (1850) ; Ressinger v. Sears Rocbuck \& Co., 62 F. Supp. 158 (N.D. Ill. 1945) ; Brill v. St. Louis Car Co., 80 Fed. 909 (E.D. Mo. 1897) ; Rein v. Clayton, 37 Fed. 354 (E.D. Mich. 1889).

The patent confers no right upon the patentee to practice the invention. Patterson v. Kentucky, 97 U.S. 501 (1878) ; In re Brosnahan, 18 Fed. 62 (W.D. Mo. 1883):

8 See Lueddecke v. Chevrolet Motor Co., 70 F.2d 345 (8th Cir. 1934).

969 C.J.S., Patents § 2 (1951) ; 42 AM. JuR., Property \$\$ 8, 815 (1942) ; Annot., 170 A.L.R. 449 (1947), 167 A.L.R. 1114 (1947). See also 5 Winliston, Contracts 4626 (1937) ; 1 Nars, Unfatr Competition and Trade Marks \$ $141 \mathrm{ff}$. (1947) ; Harris, Temporary Protection for Inventors, Los ANGejes BAR BuLtetin (April 1950), 56 CASE. \& CoM. 20 (Mar. 1951); Knoth, The Protection of Unpatented Ideas and Inventions, 32 J. PAт. OFF. Soc'x 268 (1950); Gregg, The Law of Trade Secrets in California, 24 Car. Sr. B.J. 15 (1949); Barton, $A$ Study in the Law of Trade Secrets, 13 U. CIN. L. Rev. 507 (1939); Notes, 64 Harv. L. REv. 976 (1951), 15 Geo. Wast. L. Rev. 8 (1946), 83 U. Pa. L. Rev. 367 (1935), 42 Harv. L. Rev. 254 (1928), 6 TEx. L. REv. 502 (1928), 19 CoLUM. L. Rev. 233 (1919).

10 Skoog v. McCray Refrigerator Co., 211 F.2d 254 (7th Cir. 1954); Cheney Bros. v. Doris Silk Corporation, 35 F.2d 279 (2d Cir. 1929), cert. denied, 281 U.S. 728 (1929); John H. Rice \& Co. v. Redlich Mfg. Co., 202 Fed. 155 (3rd Cir. 1913) ; John D. Park \& Sons Co. v. Hartman, 153 Fed. 24 (6th Cir. 1907), cert. granted, 206 U.S. 562 (1907), dismissed, 212 U.S. 588 (1908); William A. Meier Glass Co. v. Anchor Hocking G. Corp., 95 F.Supp. 264 (W.D.Pa. 1951); Raenore Novelties v. Superb Stitching Co., 47 N.Y.S.2d 831 (1944); Jessar Manufacturing Corporation v. Berlin, 380.Pa. 453, 110 A.2d 396 (1955) ; Chas. H. Elliott Co. v. Skillkrafters, $271 \mathrm{~Pa}$. 185, 114 Atl. 488 (1921). The cases himit this right only by precluding unnecessary copying of non-functional parts of the invention so as to confuse the purchaser as to the maker.

11 Aktiebolaget Bofors v. United States, 194 F.2d 145 (D.C. Cir. 1951); M. J. Lewis Products Co. v. Lewis, 57 F.2d 886 (E.D.Pa. 1931) ; Cheney Bros. v. Doris Silk Corporation, 35 F.2d 279 (2d Cir. 1929), cert. denied, 281 U.S. 728 (1929); Tower Mfg. Co., Inc. v. Monsanto Chemical Works, 20 F.2d 386 (S.D.N.Y. 1927); Newell v. O. A. Newton \& Son Co., 104 F. Supp. 162 (D. Del. 1952); American Stay Co. v. Delaney, 211 Mass. 229, 97 N.E. 911 (1912) ; Tabor v. Hoffman, 118 N.Y. 30, 23 N.E. 12 (1889) ; Klein v. Ekco Products Company, 135 N.Y.S.2d 391 (1954).

12 Protection is generally not dependent upon the inventor's using the invention. Ferroline Corp. v. General Aniline \& Film Corp., 207 F.2d 912 (7th Cir. 1953), cert. denied, 347 U.S. 953 (1954). But see Victor Chemical Works v. Tiff, 299 Ill. 532, 132 N.E. 806 (1921). 
matter of state law. ${ }^{13}$ The person who acquires knowledge honestly and fairly, however, is free to make, use and sell the invention without any obligation to the inventor. ${ }^{14}$ If the invention is capable of being profitably exploited without sacrificing its secret nature, the inventor may be well advised to rely on counmon law protection rather than on a patent since the former has no time limit; ${ }^{15}$ also, the common law may protect inventions which can not meet the statutory standards of patentability. ${ }^{\mathbf{1 6}}$

\section{What is a common law invention?}

The invention may be any product, process or machine which has been created by a person and which has not theretofore been part of public knowledge. ${ }^{17}$ Although such categorization is similar to that adopted for patentability, ${ }^{18}$ the strict requirements of novelty and invention found in patent law are not applied with equal stringency for common law protec-

13 Becher v. Contoure Laboratories, 279 U.S. 388 (1928) (the cause of action stems from a breach of trust or breach of contract); Franke v. Wiltschek, 209 F.2d 493 (2d Cir. 1953); Ferroline Corp. v. General Aniline \& Film Corp., 207 F.2d 912 (7th Cir. 1953), cert. denied, 347 U.S. 953 (1954); Smith v. Dravo Corp., 203 F.2d 369 (7th Cir. 1953), 208 F.2d 388 (7th Cir. 1953); Cummings v. Moore, 202 F.2d 145 (10th Cir. 1953). See, e.g., Cax. Crv. CodE $\S \S 980-984$.

A suit for infringement of a patent cannot be coupled with a suit for breach of a trade secret (unrelated to the patent) so as to get both into a federal court where the trade secret suit would not separately qualify. Newport Industries v. Crosby Naval Stores, 139 F.2d 611 (5th Cir. 1944) ; accord, Hurn v. Oursler, 289 U.S. 238 (1953).

14 Ferroline Corp. v. General Aniline \& Filun Corp., 207 F.2d 912 (7th Cir. 1953), cert. denied, 347 U.S. 953 (1954); Canfield v. Blaw-Knox Co., 98 F.2d 805 (3rd Cir. 1938); Cheney Bros. v. Doris Silk Corporation, 35 F.2d 279 (2d Cir. 1929), cert. denied, 281 U.S. 728 (1929); John H. Rice \& Co. v. Redlich Mfg. Co., 202 Fed. 155 (3rd Cir. 1913) ; John D. Park \& Sons Co. v. Hartman, 153 Fed. 24 (6th Cir. 1907), cert. granted, 206 U.S. 562 (1907), dismissed, 212 U.S. 588 (1908); Goldin v. R. J. Reynolds Tobacco Co., 22 F.Supp. 61 (S.D. N.Y. 1938); Alexis, Inc. v. Werbell, 209 Ga. 665, 75 S.E.2d 168 (1953) ; Stewart v. Hook, 118 Ga. 445, 45 S.E. 369 (1903); Pomeroy Ink Co. v. Poineroy, 77 N.J. Eq. 293, 78 Atl. 698 (1910); Petnel v. American Tel. \& Tel. Co., 280 App.Div. 706, 117 N.Y.S.2d 294 (1952) (subsequent citations omitted) ; McClary v. Hubbard, 97 Vt. 222, 122 Atl. 469 (1923).

The honest discoverer is free to advertise the fact. Jacobs v. Beecham, 221 U.S. 263 (1910); Watkins v. Landon, 52 Mimn. 389, 54 N.W. 193 (1893), 67 Mimn. 136, 69 N.W. 711 (1897).

15 American Dirigold Corp. v. Dirigold Metals Corp., 125 F.2d 446 (6th Cir. 1942) ; Nye Tool \& Machime Works v. Crown Die \& Tool Co., 276 Fed. 376 (7th Cir. 1921), rev'd on other grounds, 261 U.S. 24 (1922).

16 See note 19 infra.

17 It is doubtful if knowledge of what not to do is protected as a secret. Detachable Bit Co. v. Timken Roller Bearing Co., 133 F.2d 632 (6th Cir. 1943). See also Klivitsky, Protection of Unpatentable Ideas, 17 J. PAT. OFF. Soc'x 854 (1935).

1866 STAT. 797, 35 U.S.C. $\$ \S 100,101$ (1952). 
tion; ${ }^{10}$ however, there must be some novelty together with the secrecy, ${ }^{20}$ at least to the disclosee. ${ }^{21}$

\section{Property aspects of the unpatented invention}

The unpatented invention has frequently been regarded as property. It is assignable ${ }^{22}$ and may be licensed; ${ }^{23}$ even if not itself patentable, its transfer is adequate consideration to support a contract ${ }^{24}$ unless it infringes another's patent when practiced. ${ }^{25}$ It is often regarded as adequate considera-

19 Smith v. Dravo Corp., 203 F.2d 369 (7th Cir. 1953), 208 F.2d 388 (7th Cir. 1953) ; Sandlin v. Johnson, 141 F.2d 660 (8th Cir. 1944), 152 F.2d 8 (8th Cir. 1945); A. O. Smith Corporation v. Petroleum Iron Works Co., 73 F.2d 531 (6th Cir. 1934), modified, 74 F.2d 934 (6th Cir. 1935); International Industries v. Warren Petroleum Corp., 99 F. Supp. 907 (D. Del. 1951); Mycalex Corporation v. Pemco Corporation, 64 F. Supp. 420 (D. Md. 1946), aff'd, 159 F.2d 907 (4th Cir. 1947); Schavoir v. American Rebonded Leather Co., 104 Conn. 472, 133 Atl. 582 (1926); Sun Dial Corporation v. Rideout, 16 N.J. Super. 252, 108 A.2d 442 (1954), modified, 17 N.J. Super. 517, 111 A.2d 881 (1955) ; Fairchild Engme \& Airplane Corp. v. Cox, 50 N.Y.S.2d 643 (1944); Vulcan Detinning Co. v. Assmann, 185 App. Div. 399, 173 N.Y. Supp. 334 (1918). But cf. Canfield v. Blaw-Knox Co., 98 F.2d 805 (3rd Cir. 1938).

The trend toward progressively stricter judicial interpretation of the standard of patentable inventions during recent years, particularly by the Supreme Court, has probably increased the interest of inventors in common law protection. It is therefore pertinent to note that section 103 of the new patent code [66 Stat. 798 (1952), 35 U.S.C. $\$ 103$ (1952)] has recently been held as intended to restore the relatively liberal attitude toward invention taken by courts prior to imposition of the more recent stricter standards. Lyon v. Bausch \& Lomb Optical Co., 106 U.S.P.Q. 1, .........2d....... (2d Cir. 1955).

${ }^{20}$ Smoley v. New Jersey Zinc Co., 106 F.2d 314 (3rd Cir. 1939); Lueddecke v. Chevrolet Motor Co., 70 F.2d 345 (8th Cir. 1934); Newell v. O. A. Newton \& Son Co., 104 F. Supp. 162 (D. Del. 1952) ; Berry v. Glidden Co., 92 F. Supp. 909 (S.D. N.X. 1950) ; Plus Promotions, Inc. v. RCA Mfg. Co., Inc., 49 F.Supp. 116 (S.D.N.Y. 1943); Gerno Mfg Co. v. Combs, 209 Mo. App. 651, 240 S.W. 872 (1922); Boost Co. v. Faunce, 17 N.J. Super. 458, 86 A.2d 283 (1952); Heyden Chemical Corporation v. Burrell \& Neidig, 2 N.J. Super. 467, 64 A.2d 465 (1949); Irvington Varnish \& Insulator Co. v. Van Norde, 138 N.J. Eq. 99, 46 A.2d 201 (1946); Macbeth-Evans Glass Co. v. Schnelbach, 239 Pa. 76, 86 Atl. 688 (1913); Vann v. Toby, 260 S.W.2d 114 (Tex. Civ. App. 1953).

21 Berry v. Glidden Co., 92 F. Supp. 909 (S.D. N.Y. 1950).

If there is a contract of confidence, want of patentable invention can scarcely be a defense. Shellmar Products Co. v. Allen-Qualley Co., 36 F.2d 623 (7th Cir. 1929); Ultra-Life Laboratories v. Eames, 240 Mo. App. 851, 221 S.W.2d 224 (1949).

22 Nelson v. Commissioner of Internal Revenue, 203 F.2d 1 (6th Cir. 1953) (and the proceeds taxable) [see Fallon, The Valuation of Patents in Taxation Proceedings, 20 VA. L. REv. 539 (1934) ]; Saunders v. Commissioner of Internal Revenue, 29 F.2d 834 (3rd Cir. 1928); Ulman v. Thompson, 57 Ind. App. 126, 106 N.E. 611 (1914); Pomeroy Ink Co. v. Pomeroy, 77 N.J. Eq. 293, 78 Atl. 698 (1910); Vulcan Detinning Co. v. American Can Co., 67 N.J. Eq. 243, 58 Atl. 290 (1904) (subsequent citations omitted).

${ }^{23}$ Schnack v. Applied Arts Corporation, 283 Mich. 434, 278 N.W. 117 (1938); Ingraham v. Schaum, 157 Pa. 88, 27 Atl. 404 (1893).

24 Gas Tool Patents Corporation v. Mould, 133 F.2d 815 (7th Cir. 1943); Myers v. Gerhardt, 344 III. 620,176 N.E. 713 (1931); Burton v. Burton Stock-Car Co., 171 Mass. 437, 50 N.E. 1029 (1898).

25 Manning v. Silloway, 261 App. Div. 99, 24 N.Y.S.2d 808 (1941), affd, 286 N.X. 638, 36 N.E.2d 686 (1941). 
tion for an issue of corporate stock. ${ }^{26}$ It has been held not subject to levy by creditors, however. ${ }^{27}$

\section{The accent on secrecy}

Although the invention is often attributed characteristics of personalty, any member of the public is free to appropriate it to his own benefit once knowledge is legitimately acquired. Accordingly, common law protection of the inventor is primarily concerned with the method by which knowledge is actually acquired by one not the inventor. ${ }^{28}$ The most cited judicial description of this protection is by Justice Holmes: ${ }^{29}$

The word property as applied to ... trade secrets is an unanalyzed expression of certain secondary consequences of the primary fact that the law makes some rudimentary requirements of good faith .... The property may be denied but the consequences cannot be. Therefore the starting point for the present matter is ... that the defendant stood in confidential relations with the plaintiffs....

The protection is well described in the Restatement of Torts in its treatment of trade secrets: ${ }^{30}$

One who discloses or uses another's trade secret, without a privilege to do so, is liable to the other if

(a) he discovered the secret by improper means, or

(b) his disclosure or use constitutes a breach of confidence reposed in hin by the other in disclosing the secret to him, or

(c) he learned the secret froin a third person with notice of the facts that it was a secret and that the third person discovered it by improper means or that the third person's disclosure of it was otherwise a breach of his duty to the other, or

(d) le learned the secret with notice of the facts that it was a secret and that its disclosure was made to him by mistake.

One who learns another's trade secret from a third person without notice that it is secret and that the third person's disclosure is a breach of his duty to the other, or who learns the secret through a mistake without notice of the secrecy and the mistake,

26 Durand v. Brown, 236 Fed. 609 (6th Cir. 1916). Contra, O'Bear-Nester Glass Co. v. Antiexplo. Co., 101 Tex. 431, 108 S.W. 967, 109 S.W. 931 (1908) ("unsubstantial and shadowy right"). See Annot., 37 A.L.R.2d 918-922 (1954).

27 Chemical Foundation v. General Aniline Works, 99 F.2d 276 (3rd Cir. 1938), cert. denied, 305 U.S. 654 (1938) ; Rosenthal v. Goldstein, 112 Misc. 606, 183 N.Y. Supp. 582 (1920) [see Notes, 30 YaIe L.J. 197 (1920), 20 CorvM. L. Rev. 801 (1920), 69 U. PA. L. Rev. 65 (1920) ]. See also Notes, 81 U. PA. L. Rev. 636 (1933), 66 Sor. J. 574 (1922).

28 DuPont Powder Co. v. Masland, 244 U.S. 100 (1916); Smith v. Dravo Corp., 203 F.2d 369 (7th Cir. 1953), 208 F.2d 388 (7th Cir. 1953) ; Tower Mfg. Co., Inc. v. Monsanto Chemical Works, 20 F.2d 386 (S.D.N.Y.1927) ; Herold v. Herold China \& Pottery Co., 257 Fed. 911 (6th Cir. 1919).

29 DuPont Powder Co. v. Masiand, 244 U.S. 100, 102 (1916).

30 Restatearent, ToRts $\$ \S 757,758$ (1938). 
(a) is not liable to the other for a disclosure or use of the secret prior to the receipt of such notice, and

(b) is liable to the other for a disclosure or use of the secret after the receipt of such notice, unless prior thereto he has in good faith paid value for the secret or has so changed his position that to subject him to liability would be inequitable.

It is the employment of improper means to procure the invention, rather than its duplication per se, against which the common law protects the inventor. Any benefit from a surreptitious acquisition is, of course, precluded. ${ }^{31}$ However, the primary problem of the courts has been to decide whether a confidence has been created by an express or implied contract when someone is voluntarily given knowledge of the invention by the inventor. ${ }^{32}$ Confidential disclosures do not destroy the secrecy requisite for common law protection ${ }^{33}$ although an unconditional disclosure to even one person has been regarded to terminate it. ${ }^{34}$ It is obvious that the court has no means of knowing whether several independent inventors may be keeping secret the identical invention. If so, there would seem to be no reason why each should not have the protection of secrecy afforded a trade secret

31 Kendall et al. v. Winsor, 62 U.S. (21 How.) 322 (1858); Shaw v. Cooper, 32 U.S. (7 Pet.) 292 (1833); Aktiebolaget Bofors v. United States, 194 F.2d 145 (D.C. Cir. 1951); Tower Mfg. Co., Inc. v. Monsanto Chemical Works, 20 F.2d 386 (S.D.N.Y.1927).

32 Ferroline Corp. v. General Aniline \& Film Corp., 207 F.2d 912 (7th Cir. 1953), cert. denied, 347 U.S. 953 (1954) ; Smith v. Dravo Corp., 203 F.2d 369 (7th Cir. 1953), 208 F.2d 388 (7th Cir. 1953); Ackermans v. General Motors Corp., 202 F.2d 642 (4th Cir. 1953), cert. denied, 345'U.S. 996 (1953); Mitchell Novelty Co. v. United Mfg. Co., 199 F.2d 462 (7th Cir. 1952); Wilson v. Seng Co., 194 F.2d 399 (7th Cir. 1952); Schreyer v. Casco Products Corp., 190 F.2d 921 (2d Cir. 1951), cert. denied, 342 U.S. 913 (1952); Canfield v. Blaw-Knox Co., 98 F.2d 805 (3rd Cir. 1938); Shellmar Products Co. v. Allen-Qualley Co., 87 F.2d 104 (7th Cir. 1936), cert. denied, 301 U.S. 695 (1936); Hoeltke v. C.M. Kemp Mfg. Co., 80 F.2d 912 (4th Cir. 1935), cert. denied, 298 U.S. 673 (1935); Tower Mfg. Co., Inc. v. Monsanto Chemical Works, 20 F.2d 386 (S.D.N.Y. 1927) ; Herold v. Herold Chima \& Pottery Co., 257 Fed. 911 (6th Cir. 1919) ; International Industries v. Warren Petroleum Corp., 99 F. Supp. 907 (D. Del. 1951); DeFilippis v. Chrysler Corporation, 53 F.Supp. 977 (S.D.N.Y. 1944), aff'd, 159 F.2d 478 (2d Cir. 1947), cert denied, 331 U.S. 848 (1947); Smoley v. New Jersey Zinc Co., 24 F. Supp. 294 (D.N.J. 1938), aff d, 106 F.2d 314 (3rd Cir. 1939) ; Carver v. Harr, 132 N.J. Eq. 207, 27 A.2d 895 (1942) ; Stone v. Goss, 65 N.J. Eq. 756, 55 Atl. 736 (1903); Glass v. Kottwitz, 297 S.W. 573 (Tex. Civ. App. 1927).

33 Board of Trade v. Christie Grain \& Stock Co., 198 U.S. 236 (1904); American Dirigold Corp. v. Dirigold Metals Corp., 125 F.2d 446 (6th Cir. 1942); Pennington Engineering Co. v. Houde Engineering Corporation, 43 F. Supp. 698 (W.D. N.Y. 1941), aff'd, 136 F.2d. 210 (2d Cir. 1943), cert. denied, 320 U.S. 771 (1943) ; Radium Remedies Co. v. Weiss, 173 Minn. 342, 217 N.W. 339 (1928); Sun Dial Corporation v. Rideout, 16 N.J. Super. 252, 108 A.2d 442 (1954), modified, 17 N.J. Super. 517, 111 A.2d 881 (1955) ; Kaumagraph Co. v. Stampagraph Co., 235 N.Y. 1, 138 N.E. 485 (1923); National Starch Products v. Polymer Industries, 273 App. Div. 732, 79 N.Y.S.2d 357 (1948).

34 American Dirigold Corp. v. Dirigold Metals Corp., 125 F.2d 446 (6th Cir. 1942) ; Perkins v. Nashua Card \& Glazed Paper Co., 2 Fed. 451 (D.N.H. 1880). 
-which, according to the Restatement of Torts, need not be limited to one person's knowledge. ${ }^{35}$

An inventor frequently finds it impractical to exploit his invention alone; consequently he may disclose details to another in the course of negotiations concerning utilization or production by the other. The courts virtually unanimously regard such a relationship as confidential and prevent the disclosee from expropriating the secret unjustly for his own benefit. ${ }^{36}$ It is always advisable for the inventor to indicate in such disclosures his expectancy of compensation should the disclosee practice the invention. ${ }^{37}$ Also the inventor should be aware that the federal government has been held free to use unpatented inventions submitted to it in confidence, even after the inventor has been requested to do so, unless an express contract for compensation exists. ${ }^{38}$

Another recurring situation exists where the possessor of a secret invention must engage others to assist him in exploiting it. Such employees are held to be confidential disclosees of information acquired by them in the course of employment. ${ }^{39}$

35 Restatement, ToRTs $\$ 757$, comment $b$ (1938); Vulcan Detinning Co. v. American Can Co., 72 N.J. Eq. 387, 67 Atl. 339 (1907), 75 N.J. Eq. 542, 73 Atl. 603 (1909) (subsequent citations omitted). See Cax. Crv. Code $§ 984$.

36 Filtex Corporation v. Amen Atiyeh, 216 F.2d 443 (9th Cir. 1954); Matarese v. MooreMcCormack Lines, 158 F.2d 631 (2d Cir. 1946); Booth v. Stutz Motor Car Co. of America, 56 F.2d 962 (7th Cir. 1932); Schreyer v. Casco Products Corp., 97 F. Supp. 159 (D. Conn. 1951), afjd, 190 F.2d 921 (2d Cir. 1951), cert. denied, 342 U.S. 913 (1952); William A. Meier Glass Co. v. Anchor Hocking G. Corp., 95 F. Supp. 264 (W.D.Pa. 1951) ; Pennington Engineering Co. v. Houde Engineering Corporation, 43 F.Supp. 698 (W.D.N.Y. 1941), aff'd, 136 F.2d 210 (2d Cir. 1943), cert denied, 320 U.S. 771 (1943) ; Gilbert v. General Motors Corp., 41 F. Supp. 525 (W.D. N.Y. 1941), af'd, 133 F.2d 997 (2d Cir. 1943), cert. denied, 319 U.S. 743 (1943); Feasel v. Noxall Polish Mfg. Co., 268 Fed. 887 (E.D. Pa. 1920) ; Hollywood Motion Picture Equipment Co. v. Furer, 16 Cal.2d 184, 105 P.2d 299 (1940); Club Razor \& Blade Mfg. Corporation v. Bindzsus, 131 N.J. Eq. 283, 25 A.2d 31 (1942), aff'd, 133 N.J. Eq. 38, 30 A.2d 31 (1943); Klein v. Ekco Products Company, 135 N.Y.S.2d 391 (1954). But see Lueddecke v. Chevrolet Motor Co., 70 F.2d 345 (8th Cir. 1934). But cf. Kelly-Koett Mfg. Co. v. McEuen, 130 F.2d 488 (6th Cir. 1942), cert. denied, 318 U.S. 762 (1942). See Gomory, Problems Arising on Submission to Corporations of Patentable or Non-Patentable Ideas or Inventions, 28 J. PAT. OrF. Soc'x 401 (1946).

37 Plus Promotions, Inc. v. RCA Mfg. Co., Inc., 49 F. Supp. 116 (S.D. N.Y. 1943).

${ }^{38}$ Gearon v. United States, 115 F. Supp. 910 (Ct. Cl. 1953), 121 F. Supp. 652 (Ct. Cl. 1954), cert. denied, 348 U.S. 942 (1955); Patton v. United States, 75 F. Supp. 470 (Ct. Cl. 1948).

30 Becher v. Contoure Laboratories, 279 U.S. 388 (1928) ; DuPont Powder Co. v. Masland, 244 U.S. 100 (1916) ; Union Carbide and Carbon Corp. v. Stuart Laboratories, 194 F.2d 823 (3rd Cir. 1952), affirming, 97 F. Supp. 713 (D. N.J. 1951); Harrison v. Glucose Sugar Refining Co., 116 Fed. 304 (7th Cir. 1902) ; C. F. Simunons Medicine Co. v. Simmons, 81 Fed. 163 (E.D. Ark. 1897); Riess v. Sanford, 47 Cal.App.2d 244, 117 P.2d 694 (1941); Germo Mfg. Co. v. McClelland, 107 Cal.App. 532, 290 Pac. 534 (1930); Julius Hyman \& Co. v. Velsicol Corp., 123 Colo. 563, 233 P.2d 977 (1951), cert. denied, 342 U.S. 870 (1951) ; Westervelt v. National Paper \& Supply Co., 154 Ind. 673, 57 N.E. 552 (1900); Junker v. Plummer, 320 Mass. 76, 67 N.E.2d 667 (1946); American Stay Co. v. Delaney, 211 Mass. 229, 97 N.E. 911 (1912); Peabody v. Norfolk, 98 Mass. 452, 96 Am. Dec. 664 (1868); Glucol Mfg. Co. v. Schulist, 239 
A confidential relationship has also been found to exist between joint inventors. ${ }^{40}$

\section{Remedies}

Equity protects the inventor's secrecy. ${ }^{41}$ Accordingly, the person who acquires knowledge improperly or who violates a confidence can be enjoined from practicing the invention or from disclosing it to others. ${ }^{42}$ Likewise, equity will require an accounting of the profits earned by the disclosee's practice of the invention. ${ }^{43}$ In addition, the disclosee may be required to pay

Mich. 70, 214 N.W. 152 (1927) ; O. \& W. Thum Co. v. Tloczynski, 114 Mich. 149, 72 N.W. 140 (1897) (excellent review of cases through 1897); Luckett v. Orange Julep Co., 271 Mo. 289, 196 S.W. 740 (1917) ; A. Hollander \& Son v. Imperial Fur Blending Corp., 2 N.J. Super. 235, 66 A.2d 319 (1949); Cameron Mach. Co. v. Samuel M. Langston, 115 Atl. 212 (N.J. Ch. 1921); Taylor Iron \& Steel Co. v. Nichols, 73 N.J.Eq. 684, 69 Atl. 186 (1908); Salomon v. Hertz, 40 N.J. Eq. 400, 2 Atl. 379 (1886); Clark Paper \& Mfg. Co. Stenacher, 236 N.Y. 312 , 140 N.E. 708 (1923) ; Kaumagraph Co. v. Stampagraph Co., 235 N.Y. 1, 138 N.E. 485 (1923); Lamont, Corliss \& Co. v. Bonnie Blend Choc. Corp., 135 Misc. 537, 238 N.Y. Supp. 78 (1929); Applebce v. Skiwanek, 27 N.Y. Crim. 78, 140 N.Y. Supp. 450 (1912) ; Perfect Measuring Tape Co. v. Notheis, 93 Ohio App. 507, 114 N.E.2d 149 (1953); Belmont Laboratories v. Heist, 303 Pa. 7, 154 Atl. 19 (1931); Macbeth-Evans Glass Co. v. Schnelbach, 239 Pa. 76, 86 Atl. 688 (1913); Fratich v. Despar, 165 Pa. 24, 30 Atl. 521 (1894). But cf. Newell v. O. A. Newton \& Son Co., 104 F. Supp. 162 (D. Del. 1952); King v. Gannon, 261 Mass. 94, 158 N.E. 346 (1927). See Note, 1 Wyo. L.J. 150 (1947); RestatenaENT, AGENCY \$\$ 395, 396 (1933).

40 Saco-Lowell Shops v. Reynolds, 141 F.2d 587 (4th Cir. 1944).

41 Hoeltke v. C. M. Kemp Mfg. Co., 80 F.2d 912 (4th Cir. 1935), cert. denied, 298 U.S. 673 (1935) ; Newell v. O. A. Newton \& Son Co., 104 F. Supp. 162 (D. Del. 1952) ; Pennington Engineering Co. v. Houde Engineering Corporation, 43 F.Supp. 698 (W.D.N.Y. 1941), aff'd, 136 F.2d 210 (2d Cir. 1943), cert. denied, 320 U.S. 771 (1943); Holley v. Hunt, 13 Cal.App.2d 335, 56 P.2d 1240 (1936); Larx Co. v. Nicol, 224 Minn. 1, 28 N.W.2d'705 (1946); Harrington v. National Outdoor Advertising Co., 335 Mo. 524, 196 S.W.2d 786 (1946) ; Spiselman v. Rabinowitz, 270 App. Div. 548, 61 N.Y.S.2d 138 (1946) ; Perfect Measuring Tape Co. v. Notheis, 93 Ohio App. 507, 114 N.E.2d 149 (1953).

42 Internat'l News Serv. v. Asso. Press, 248 U.S. 215 (1918) ; DuPont Powder Co. v. Masland, 244 U.S. 100 (1916); Sandlin v. Johnson, 141 F.2d 660 (8th Cir. 1944), 152 F.2d 8 (8th Cir. 1945) ; American Dirigold Corp. v. Dirigold Metals Corp., 125 F.2d 446 (6th Cir. 1942); Elaterite Paint \& Mfg. Co. v. S. E. Frost Co., 105 Mimn. 239, 117 N.W. 388 (1908); Cameron Mach. Co. v. Samuel M. Langston, 115 Atl. 212 (N.J. Ch. 1921); McKenzie v. Clime, 197 Ore. 184, 252 P.2d 564 (1953); Simmons Hardware Co. v. Waibel, I S.D. 488, 47 N.W. 814 (1891).

The remedy of injunction inay be mappropriate once a disclosure has been made to the public. Schreyer v. Casco Products Corp., 190 F.2d 921 (2d Cir. 1951), cert. denied, 342 U.S. 913 (1952) ; Conmar Products Corp. v. Universal Slide Fastener Co., 172 F.2d 150 (2d Cir. 1949).

43 Ackermans v. General Motors Corp., 202 F.2d 642 (4th Cir. 1953), cert. denied, 345 U.S. 996 (1953) ; Schreyer v. Casco Products Corp., 190 F.2d 921 (2d Cir. 1951), cert. denied, 342 U.S. 913 (1952); Conmar Products Corp. v. Universal Shide Fastener Co., 172 F.2d 150 (2d Cir. 1949); Sandlin v. Johnson, 141 F.2d 660 (8th Cir. 1944), 152 F.2d 8 (8th Cir. 1945); SacoLowell Shops v. Reynolds, 141 F.2d 587 (4th Cir. 1944); Hoeltke v. C. M. Kemp Mfg. Co., 80 F.2d 912 (4th Cir. 1935), cert. denied, 298 U.S. 673 (1935); Irving Iron Works v. Kerlow Steel Flooring Co., 103 N.J. Eq. 240, 143 Atl. 145 (1926) ; McKinzie v. Cline, 197 Ore. 184, 252 P.2d 564 (1953). 
damages to the inventor for harm resulting from the disclosee's misuse of his knowledge. ${ }^{44}$

Third parties acquiring knowledge from the breaching disclosee are similarly liable to the inventor for disclosure or use from the time at which they acquire notice that the invention was secret and wrongfully obtained from the inventor. ${ }^{45}$ However, if the third party has paid value for his information or has materially changed his position in reliance thereon before receiving such notice, he may be allowed to continue practicing the invention without obligation. ${ }^{46}$ The innocently acquiring third party may in turn be able to obtain relief against improper acquisition of the secret from him. ${ }^{47}$

Any unchallenged public disclosure of the invention obviously destroys the secrecy upon which the inventor's common law protection is based.8 Sale or exhibition of a product may be a disclosure of the product itself $f^{4 \theta}$ but it need not be a disclosure of the process or machine which made the product, at least, if done by someone other than the inventor. ${ }^{50} \mathrm{~A}$ public

44 Ackermans v. General Motors Corp., 202 F.2d 642 (4th Cir. 1953), cert. denied, 345 U.S. 996 (1953); Schreyer v. Casco Products Corp., 190 F.2d 921 (2d Cir. 1951), cert. denied, 342 U.S. 913 (1952) ; Conmar Products Corp. v. Universal Slide Fastener Co., 172 F.2d 150 (2d Cir. 1949); Sandlin v. Johnson, 141 F.2d 660 (8th Cir. 1944), 152 F.2d 8 (8th Cir. 1945); SacoLowell Shops v. Reynolds, 141 F.2d 587 (4th Cir. 1944); Hoeltke v. C. M. Kemp Mfg. Co., 80 F.2d 912 (4th Cir. 1935), cert. denied, 298 U.S. 673 (1935) ; Michel Cosmetics, Inc. v. Tsirkas, 282 N.Y. 195, 26 N.E.2d 16 (1940) ; Brown \& Root v. Jacques, 98 S.W.2d 257 (Tex. Civ. App. 1936).

45 Conmar Products Corp. v. Universal Stide Fastener Co., 172 F.2d 150 (2d Cir. 1949); Herold v. Herold China \& Pottery Co., 257 Fed. 911 (6th Cir. 1919) ; Mycalex Corporation v. Pemco Corporation, 64 F. Supp. 420 (D.Md. 1946), affd, 159 F.2d 907 (4th Cir. 1947); Westervelt v. National Paper \& Supply Co., 154 Ind. 673, 57 N.E. 552 (1900); Flexmir, Inc. v. Herman, 138 N.J.Eq. 594, 49 A.2d 489 (1946); Vulcan Detinning Co. v. American Can Co., 75 N.J. Eq. 542, 73 Atl. 603 (1909) (subsequent citations omitted) ; Stone v. Goss, 65 N.J. Eq. 756, 55 Atl. 736 (1903); Macbeth-Evans Glass Co. v. Schnelbach, 239 Pa. 76, 86 Atl. 688 (1913). Cf. Stewart v. Hook, 118 Ga. 445, 45 S.E. 369 (1903).

46 Conmar Products Corp. v. Universal Slide Fastener Co., 172 F.2d 150 (2d Cir. 1949); Mycalex Corporation v. Penico Corporation, 64 F. Supp. 420 (D.Md.1946), aff'd, 159 F.2d 907 (4th Cir. 1947). Cf. Stewart v. Hook, 118 Ga. 445, 45 S.E. 369 (1903) [see Note, 17 Harv. L. REv. 206 (1904)].

47 Vulcan Detinning Co. v. American Can Co., 72 N.J. Eq. 387, 67 Atl. 339 (1907), 75 N.J. Eq. 542, 73 Atl. 603 (1909) (subsequent citations omitted).

48 Kendall et al. v. Winsor, 62 U.S. (21 How.) 322 (1858) ; Shaw v. Cooper, 32 U.S. (7 Pet.) 292 (1833); Sandlin v. Johnson, 141 F.2d 660 (8th Cir. 1944), 152 F.2d 8 (8th Cir. 1945).

49 Northup v. Reish, 200 F.2d 924 (7th Cir. 1953); Cheney Bros. v. Doris Silk Corporation, 35 F.2d 279 (2d Cir. 1929), cert. denied, 281 U.S. 728 (1930); Newell v. O. A. Newton \& Co., 104 F.Supp. 162 (D.Del. 1952); Willian A. Meier Glass Co. v. Anchor Hocking G. Corp., 95 F.Supp. 264 (W.D.Pa. 1951) ; Carver v. Harr, 132 N.J. Eq. 207, 27 A.2d 895 (1942); Wissman v. Boucher, 150 Tex. 326, 240 S.W.2d 278 (1951); Brown \& Root v. Jacques, 98 S.W.2d 257 (Tex. Civ. App. 1936).

50 Metallizing Engineer Co. v. Kenyon Bearing \& A. P. Co., 153 F.2d 516 (2d Cir. 1946), cert. denied, 328 U.S. 840 (1946), overruling, Peerless Roll Leaf Co. v. H. Griffin \& Sons Co:, 29 F.2d 646 (2d Cir. 1928) ; Gillman v. Stern, 114 F.2d 28 (2d Cir. 1940), cert. denied, 311 U.S. 718 (1941); Tabor v. Hoffinan, 118 N.Y. 30, 23 N.E. 12 (1889). 
use of an invention by one person is enough to destroy secrecy. ${ }^{51}$ Some inventions by nature are concealed from the public eye while in use-nevertheless, the use may be public if no restriction of secrecy is put upon the user. ${ }^{52}$ A patent grant, but not a patent application, is also a public disclosure which terminates the common law protection. ${ }^{53}$ Any public disclosure is, of course, irrevocable..$^{54}$

The public is free to practice the invention after a general disclosure although naturally risking infringement action if the disclosure has been by patenting. Such disclosure does not affect a cause of action arising from a previous breach of confidence by the disclosee, ${ }^{65}$ even if disclosure has been by a patent and the breach, if continued, would not constitute infringement. ${ }^{56}$ The disclosee who has acquired knowledge first in confidence, however, may be precluded not only from benefiting from any subsequent investigations which he might conduct to legitimately uncover the secret ${ }^{57}$ but also, on occasion, from benefiting by practice of the invention after general disclosure to him as a member of the public. ${ }^{58}$ If licensed by the

51 Egbert v. Lippman, 104 U.S. 333 (1881) ; Skoog v. McCray Refrigerator Co., 211 F.2d 254 (7th Cir. 1954). In the Skoog case, common law protection was denied the inventor of a display-type refrigeration cabinet because he had utilized the invention in his store suhject to unrestricted public use for two months prior to making a confidential disclosure to the defendant. As an alternative ground, the court held that the defendant had independently invented the same device.

52 Egbert v. Lippman, 104 U.S. 333 (1881).

53 Sandlin v. Johnson, 141 F.2d 660 (8th Cir. 1944), 152 F.2d 8 (8th Cir. 1945); A. O. Smith Corporation v. Petroleum Iron Works Co., 73 F.2d 531 (6th Cir. 1934), modified, 74 F.2d 934 (6th Cir. 1935); Darsyn Laboratories v. Lenox Laboratories, 120 F. Supp. 42 (D. N.J. 1954), aff'd, 217 F.2d 648 (3rd Cir. 1954) ; Julius Hyman \& Co. v. Velsicol Corp., 123 Colo. 563, 233 P.2d 977 (1951), cert. denied, 342 U.S. 870 (1951).

54 Pennock v. Dialogue, 27 U.S. (2 Pet.) 1 (1829) ; Smith v. Dravo Corp., 203 F.2d 369 (7th Cir. 1953), 208 F.2d 388 (7th Cir. 1953). See note 70 infra.

55 Wilson v. Seng Co., 194 F.2d 399 (7th Cir. 1952); Picard v. United Aircraft Corporation, 128 F.2d 632 (2d Cir. 1942), cert. denied, 317 U.S. 651 (1942) ; Gilbert v. General Motors Corporation, 32 F.Supp. 502 (W.D. N.Y. 1940), af'd, 133 F.2d 997 (2d Cir. 1943), cert. denied, 319 U.S. 743 (1943).

If the disclosure hy patenting is followed by a hicensing of the disclosee, the post-patent royalty arrangement may set the measure of recovery for the pre-patent breach of confidence. Kelley-Koett Mfg. Co. v. McEuen, 130 F.2d 488 (6th Cir. 1942), cert. denied, 318 U.S. 762 (1943).

56 Wilson v. Seng Co., 194 F.2d 399 (7th Cir. 1952) ; Gilbert v. General Motors Corporation, 32 F. Supp. 502 (W.D. N.Y. 1940), aff'd, 133 F.2d 997 (2d Cir. 1943), cert. denied, 319 U.S. 743 (1943).

67 Franke v. Wiltschek, 209 F.2d 493 (2d Cir. 1953); Shellmar Products Co. v. AllenQualley Co., 36 F.2d 623 (7th Cir. 1929); Stone v. Goss, 65 N.J.Eq. 756, 55 Atl. 736 (1903).

58 Shellmar Products Co. v. Allen-Qualley Co., 87 F.2d 104 (7th Cir. 1936), cert. denied, 301 U.S. 695 (1937) ; A. O. Smith Corporation v. Petroleuin Iron Works Co., 74 F.2d 934 (6th Cir. 1934). Contra, Northup v. Reish, 200 F.2d 924 (7th Cir. 1953); Schreyer v. Casco Products Corp., 190 F.2d 921 (2d Cir. 1951), cert. denied, 342 U.S. 913 (1952) ; Conmar Products Corp. v. Universal Shde Fastener Co., 172 F.2d 150 (2d Cir. 1949) ; Sandlin v. Johnson, 141 F.2d 660 (8th Cir. 1944), 152 F.2d 8 (8th Cir. 1945) ; Picard v. United Aircraft Corpora- 
inventor before disclosure, he may be required not only to make payment of royalties due before disclosure but also to respect the terms of an existing license after disclosure. ${ }^{59}$

\section{Effect of the selection of either common law or patent protection}

The common law, stressing secrecy, and the patent statutes, stressing disclosure, are mutually inconsistent, at least to a considerable extent. Although a patent application is not destructive of secrecy, the issued patent contains the patentee's full and voluntary public disclosure. ${ }^{60}$ Accordingly, common law protection is thereafter unavailable, even though the patent may ultimately prove void. ${ }^{61}$

The effect of secret exploitation upon subsequent patentability $b y$ the exploiter, on the other hand, is not so clearly defined. Certain of the technical statutory requirements for patentability concern dispositions made of the invention prior to application. ${ }^{62}$ The period of exclusiveness accorded

tion, 128 F.2d 632 (2d Cir. 1942), cert. denied, 317 U.S. 651 (1942); Flanigan v. Ditto, Inc., 84 F.2d 490 (7th Cir. 1936), cert. denied, 299 U.S. 598 (1936); Carver v. Harr, 132 N.J. Eq. 207, 27 A.2d 895 (1942). But cf. William A. Meier Glass Co. v. Anchor Hocking G. Corp., 95 F.Supp. 264 (W.D.Pa. 1951); Berry v. Glidden Co., 92 F.Supp. 909 (S.D. N.Y. 1950); Fairchild Engine \& Airplane Corp. v. Cox, 50 N.Y.S.2d 643 (1944).

60 Sandlin v. Johnson, 141 F.2d 660 (8th Cir. 1944), 152 F.2d 8 (8th Cir. 1945); Carver v. Harr, 132 N.J. Eq. 207, 27 A.2d 895 (1942).

If the inventor's disclosure was wrongful as to the licensees, they may have a cross-complaint against him. American ChlorophylI v. Schertz, 176 Va. 362, 11 S.E.2d 625 (1940),

60 See note 53 supra.

61 See text at notes 53 supra, 70 infra. Nor will equity protect the "best method" of practicing the invention if this has been retained in secrecy instead of disclosed in the patent application. Dow Chemical Co. v. American Bromine Co., 210 Mich. 262, 177 N.W. 996 (1920); cf. Union Carbide and Carbon Corp. v. Stuart Laboratories, 194 F.2d 823 (3rd Cir. 1952), cert. denied, 343 U.S. 967 (1952).

6266 STAT. 797-798 (1952), 35 U.S.C. $\$ 102$ (1952): "Conditions for patentability; novelty and loss of right to patent

"A person shall be entitled to a patent unless-

"(a) the invention was known or used by others in this country, or patented or described in a printed publication in this or a foreign country, before the invention thereof by the applicant for patent, or

"(b) the invention was patented or described in a printed pubhication im this or a foreign country or in public use or on sale in this country, more than one year prior to the date of the application for patent in the United States, or

"(c) he has abandoned the invention, or

"(d) the invention was first patented or caused to be patented by the applicant or his legal representatives or assigns in a foreign country prior to the date of the application for patent in this country on an application filed more than twelve months before the filing of the application in the United States, or

"(e) the invention was described in a patent granted on an application for patent by another filed in the Umited States before the invention thereof by the applicant for patent, or

"(f) he did not himself imvent the subject matter sought to be patented, or

"(g) before the applicant's invention thereof the invention was made in this country by another who had not abandoned, suppressed, or concealed it. In determining priority of invention there shall be considered not only the respective dates of conception and reduction to practice of the invention, but also the reasonable diligence of one who was first to conceive and last to reduce to practice, from a time prior to conception by the other." 
by a patent is for seventeen years; consequently, it is to be expected that the courts will view with disfavor any attempt to prolong this period. In: deed, the courts have often stated that the inventor's election to exploit the invention under secrecy will constitute a bar to his subsequent acquisition of a patent. The bar has been defined as resulting from "laches," "abandonment of the invention" or "forfeiture." 63 At least one year's secret use, however, should not be a bar inasmuch as one year's public use is not..$^{04}$

A collateral question concerns patenting of an invention by a second independent inventor when the first inventor has elected to use secrecy in obtaining exclusivity during exploitation. ${ }^{65}$ Several early cases, in accord with 35 U.S.C. $\$ 102 \mathrm{a},{ }^{66}$ have indicated that secret knowledge or use of an invention in this country by a prior but contemporaneous inventor who still retains knowledge might preclude a patent on the invention by a subsequent independent inventor. ${ }^{67}$ However, subsection 35 U.S.C. $\$ 102 \mathrm{~g}$ now expressly provides that "suppression or concealment" of the invention by a first inventor will not be grounds for denying a patent to the second inventor and modern cases have advocated the same conclusion. ${ }^{63}$ Under this

${ }^{63}$ Woodbridge v. United States, 263 U.S. 50 (1923); Kendall et al. v. Winsor, 62 U.S. (21 How.) 322 (1858) ; Pennock v. Dialogue, 27 U.S. (2 Pet.) 1 (1829); Huszar v. Cincinnati Chemical Works, 172 F.2d 6 (6th Cir. 1949); Metallizing Engineer Co. v. Kenyon Bearing \& A.P. Co., 153 F.2d 516 (2d Cir. 1946), cert. denied, 328 U.S. 840 (1946); Allinson Mfg. Co. v. Ideal Filter Co., 21 F.2d 22 (8th Cir. 1927); Victor Talking Mach. Co. v. Starr Piano Co., 281 Fed. 60 (2d Cir. 1922), cert. denied, 260 U.S. 726 (1922); Macbeth-Evans Glass Co. v. General Electric Co., 246 Fed. 695 (6th Cir. 1917), cert. denied, 246 U.S. 659 (1918) [see Note, 31 HARv. L. Rev. 1033 (1918) ]; E. W. Bliss Co. v. Southern Can Co., 251 Fed. 903 (D. Md. 1918), af'd, 265 Fed. 1018 (4th Cir. 1920). Cf. Wirebounds Patents Co. v. Saranac Automatic Mach. Corp., 65 F.2d 904 (6th Cir. 1933); Miller v. Hayınan, 46 F.2d 188 (C.C.P.A. 1931); Vanore v. Improta, 25 F.2d 918 (D.C. Cir. 1928); Doulett v. Muther, 277 Fed. 600 (D.C. Cir. 1922). But see Bates v. Coe, 98 U.S. (8 Otto) 31 (1878).

Experimental use in perfecting the invention is not regarded as counmercial exploitation. Agawam Company v. Jordan, 74 U.S. (7 Wall.) 583 (1868); Kendall et al. v. Winsor, 62 U.S. (21 How.) 322 (1858).

The stigma of pre-patent secrecy is aggravated where application is ultimately made in fear of disclosure or competition by another. Kendall et al. v. Winsor, 62 U.S. (21 How.) 322 (1858); Stresau v. Ipsen, 77 F.2d 937 (C.C.P.A. 1935); E. W. Bliss Co. v. Southern Can Co., 251 Fed. 903 (D. Md. 1918), affd, 265 Fed. 1018 (4th Cir. 1920).

6466 STAT. 797 (1952), 35 U.S.C. §102b (1952); Metallizing Engineer Co. v. Kenyon

Bearing \& A. P. Co., 153 F.2d 516 (2d Cir. 1946), cert. denied, 328 U.S. 840 (1946).

65 See Ellis, Subsequent Inventor's Patent Rights With Regard to an Invention Previously

Made by Another Who Kept It Secret, 35 J. Pat. OFF. Soc'y 259 (1953).

66 See note 62 supra.

${ }^{67}$ Coffin v. Ogden, 85 U.S. (18 Wall.) 120 (1873); Gayler et al. v. Wilder, 51 U.S.

(10 How.) 477 (1850).

08 Milburn Co. v. Davis etc. Co., 270 U.S. 390 (1926); Gillman v. Stern, 114 F.2d 28

(2d Cir. 1940); E. W. Bliss Co. v. Southern Can Co., 251 Fed. 903 (D.Md. 1918), affd, 265

Fed. 1018 (4th Cir. 1920); Acme Flexible Clasp Co. v. Cary Mfg. Co., 96 Fed. 344 (S.D.N.Y. 1899), aff d, 101 Fed. 269 (2d Cir. 1900), 108 Fed. 873 (2d Cir. 1901), dismissed, 187 U.S. 427 (1903); cf. Bates v. Coe, 98 U.S. (8 Otto) 31 (1878).

The logical judicial attitude that secret knowledge by a prior inventor should not act as a 
view the inventor who relies upon secrecy could later find himself infringing a patent on his own invention in the hands of another. No case yet appears to have reached such a consequence. ${ }^{69}$

\section{Patenting of the invention by a person other than the inventor}

A particularly difficult problem arises when the confidential disclosee has proceeded to obtain a patent for himself upon the invention of another. By the patent issue the invention theoretically has been completely disclosed and secrecy irrevocably destroyed.$^{70}$ Accordingly, general use by the public would seemingly be subject, at most, to the patentee's right of exclusion. ${ }^{71}$ However, under the patent statutes the patent is unquestionably void by virtue of having been granted to a person not the true inventor; therefore it conveys no rights as against the public. ${ }^{72}$ What remedy exists for the inventor in such a situation?

In Kennedy v. Hazelton ${ }^{73}$ the Supreme Court stated that equity will not compel the assignment of a void patent, since the only purpose that could be served thereby would be an unjustified imposition upon the public rights. Nor could an accounting for profits received under the patent be maintained since there is neither legal nor equitable ownership of a void patent. ${ }^{74}$

In Becher v. Contoure Laboratories ${ }^{75}$ the confidential disclosee had patented the invention as his own. The inventor had then obtained a decree

patent bar to a subsequent and independent invention appears to be filatly contradicted by Congress as to inventions covered by the Atomic Energy Act of 1954. 68 STAT. 947 (1954), 42 U.S.C.A. § 2185 (Supp. 1954).

For a comparison with the solutions adopted in other countries, see Riesenfeld, The New American Patent Act in the Light of Comparative Law, 102 U. PA. L. REv. 291 (1954), 102 U. PA. L. Rev. 723 (1954) ; e.g., the German Act gives priority to the first applicant for patent.

69 This discussion is not concerned, of course, with prior public use-which precludes common law protection entirely, which precludes patenting by a subsequent "imventor" and which precludes patenting by the first inventor if persisted in for over one year prior to application.

70 Smith v. Dravo Corp., 203 F.2d 369 (7th Cir. 1953), 208 F.2d 388 (7th Cir. 1953); Conmar Products Corp. v Umiversal Slide Fastener Co., 172 F.2d 150 (2d Cir. 1949) (even though patent eventually proves invalid); Sandlin v. Johnson, 141 F.2d 660 (8th Cir. 1944), 152 F.2d 8 (8th Cir. 1945); Darsyn Laboratories v. Lenox Laboratories, 120 F. Supp. 42 (D. N.J. 1954), affd, 217 F.2d 648 (3rd Cir. 1954).

71 No case has suggested an ability of the inventor to regard the public as acquiring knowledge gratuitously from the disclosee's breach of confidence, thereby enabling the inventor to seek the remedies as set forth in text at note 45 supre.

7266 StaT. 798, 799 (1952), 35 U.S.C. $\$ \S 111,115$ (1952) ; Kennedy v. Hazelton, 128 U.S. 667 (1888).

73128 U.S. 667 (1888).

74 Ibid.; Friedman v. Washburn Co., 155 F.2d 959 (7th Cir. 1946). But cf. Bohlman v. American Paper Goods Co., 66 F.Supp. 828 (D.N.J. 1946); Murjahn v. Hall, 119 Fed. 186 (S.D. N.Y.1902).

75279 U.S. 388 (1928). 
from a state court declaring the disclosee a trustee ex maleficio of the invention and the patent, ordering him to assign the patent to the inventor, and forbidding him to practice the invention. The disclosee then proceeded to bring a patent infringement suit against the inventor in a federal court. The Supreme Court, noting that the jurisdiction of federal courts is exclusive in cases arising under the patent laws, held that the inventor's suit in the state court arose not under patent law but under a non-federal cause of action for breach of contract or wrongful disregard of confidential relations. ${ }^{76}$ Although the patent was obviously void, the Court nevertheless held that the decreed assignment at least served to estop the disclosee from bringing an infringement suit against the inventor. The effect of the Becher decision upon the Kennedy doctrine has been a subject of hopeless dispute. ${ }^{77}$

The lower federal courts have adhered to the view that it would be error to order assignment of a void patent obtained by the disclosee or to order an accounting for profits received under such patent by the disclosee although other relief outside of patent law may be awarded. ${ }^{78}$ This relief may be damages resulting from the public disclosure, an accounting for profits from practice of the invention by the disclosee and an injunction precluding such practice in the future, at least by the disclosee; ${ }^{70}$ such relief is that commonly given for a breach of confidence. However, a suit to void or cancel such a patent can be brought solely by the United States. ${ }^{80}$ The inventor may challenge the patent only by applying and qualifying for a patent himself, whereby the effect of the disclosee's patent could be viti-

76 Becher v. Contoure Laboratories, 279 U.S. 388 (1928) ; Eckert v. Braun, 155 F.2d 517 (7th Cir. 1946); Hoeltke v. C. M. Kemp Mfg. Co., 80 F.2d 912 (4th Cir. 1935), cert denied, 298 U.S. 673 (1935); Miller v. Rudolph Wurlitzer Co., 48 F. Supp. 772 (W.D. N.Y. 1942).

77 See Riesenfeld, The New American Patent Act in the Light of Comparative Law, $102 \mathrm{U}$. PA. L. Rev. 723, 751 n.191 (1954). The guarded language in the Becher decision makes it appear most unlikely that any reconsideration of the Kennedy doctrine was at issue.

78 Friedman v. Washburn Co., 145 F.2d 715 (7th Cir. 1944), 155 F.2d 959 (7th Cir. 1946); Crook v. Bendix Aviation Corporation, 68 F.Supp. 449 (D. Del. 1946) ; Miller v. Rudolph Wurhitzer Co., 48 F.Supp. 772 (W.D.N.Y. 1942); Liquid Carbonic Corp. v. Goodyear Tire \& Rubber Co., 38 F.Supp. 520 (N.D. Ohio 1940). But cf. Bohlman v. American Paper Goods Co., 66 F.Supp. 828 (D.N.J. 1946) ; Murjahn v. Hall, 119 Fed. 186 (S.D.N.Y. 1902).

70 Friedman v. Washburn Co., 155 F.2d 959 (7th Cir. 1946); Hoeltke v. C. M. Kemp Mfg. Co., 80 F.2d 912 (4th Cir. 1935), cert. denied, 298 U.S. 673 (1935) ; Murjahn v. Hall, 119 Fed. 186 (S.D.N.Y. 1902). A distinction must be recognized between an accounting for profits from the disclosee's practice of the invention and an accounting for profits received by the disclosec under the patent, i.e., from licensing others.

80 Briggs v. United Shoe Co., 239 U.S. 48 (1915); United States v. Bell Telephone Co., 128 U.S. 315 (1888); Eckert v. Braun, 155 F.2d 517 (7th Cir. 1946). 
ated after interference proceedings in the Patent Office, ${ }^{81}$ or by defending an infringement action. ${ }^{82}$

Some state courts have adhered to the approach of the state court in the Becher case and have decreed that the disclosee is a trustee ex maleficio of the patent and must account for profits obtained thereunder; ${ }^{83}$ others adopt the approach of the federal courts. ${ }^{84}$

Thus the remedies for an inventor whose invention has been patented by another in breach of confidence are of limited scope. The patent itself is void and useless whether or not assigned to him; ${ }^{85}$ in some state courts the inventor can obtain an accounting of any profits received under the patent by the disclosee. Although further practice by the disclosee may be enjoined, no ability to exclude the public has been suggested and the inventor is apparently restricted to recourse against the disclosee for damages resulting from the loss of secrecy.

It may be interesting to note that the German patent law specifically provides for the protection of the inventor whose invention has been illegally appropriated by another and made the subject of the hostile patent application or patent. The damaged inventor may either demand the assignment of the patent or the right to the patent or may file an opposition to the patent application of the appropriator or may demand cancellation of the patent..$^{88}$

\section{Preservation of the inventor's secrecy during litigation}

In cases where an inventor seeks judicial aid for the protection of his unpatented invention, it is obvious that any need for knowledge of the invention by the litigants and the court for facilitating the trial is incompatible with the inventor's interest in secrecy. There is no justification for requiring the secret to be divulged where the information is not necessary

8166 STAT. 801-802, 814 (1952), 35 U.S.C. \$§ 135, 291 (1952); Hise v. Grasty, 159 Va. 535, 166 S.E. 567 (1932).

8266 STAT. 812 (1952), 35 U.S.C. $\$ 281$ (1952). An alleged infringer may also bring a declaratory judgment action against the patentee, asking that the patent be declared invalid. Cummings v. Moore, 202 F.2d 145 (10th Cir. 1953) ; E. Edelmann \& Co. v. Triple-A Specialty Co., 88 F.2d 852 (7th Cir. 1937), cert. denied, 300 U.S. 680 (1937).

83 Irving Iron Works v. Kerlow Steel Flooring Co., 96 N.J. Eq. 702, 126 Atl. 291 (1924), 103 N.J.Eq. 240, 143 Atl. 145 (1926); Mayer v. Hochman, 98 N.Y.S.2d 724 (1950).

84 New Era Electric Range Co. v. Serrell, 252 N.Y. 107,169 N.E. 105 (1929); Hise v. Grasty, 159 Va. 535, 166 S.E. 567 (1932) [see Note, 81 U.PA.L. REv. 636 (1933)].

85 The instant situation should not be confused with that where the defendant has acquired a valid patent which equity regards another as entitled to. Shellmar Products Co. v. AllenQualley Co., 36 F.2d 623 (7th Cir. 1929).

86 German Patent Law of May 5, $1936 \$ \$ 4(3), 5,13(1)(3)$; see Riesenfeld, The New United States Patent Act in the Light of Comparative Law, 102 U.PA.L. REv. 723,751 n.191 (1954). 
or relevant. ${ }^{87}$ However, if it becames apparent, either before ${ }^{88}$ or during the trial, that disclosure of the invention is essential to further proceedings, the disclosure can be compelled. ${ }^{80}$

The court's discretion must be relied upon to limit the degree of disclosure to the minimum commensurate with a fair trial of the case; ${ }^{00} i . e$. , in cases involving secret preparations, a qualitative disclosure of ingredients may suffice without insistence upon a quantitative disclosure. ${ }^{01}$

When disclosure of the invention is unavoidable, it should be accomplished in a manner designed to safeguard the secret to the greatest extent feasible. ${ }^{92}$ If disclosure is to be by extra-trial discovery and interrogation, it may be made by conducting a private examination before a court officer and the litigants ${ }^{\mathbf{9 3}}$ instead of requiring disclosure by a bill of particulars. ${ }^{04}$ Preservation of the secret during trial may be furthered by receiving testimony at the trial in camera, restricting presentation of such evidence on appeal to members of the courts only and eliminating any description thereof in publislied opinions. ${ }^{85}$

\footnotetext{
878 WraMore, EvIDENCE § 2212 (3rd ed. 1940); Annot., 17 A.L.R. 2d 383, 388 (1951).

88 For a compilation and discussion of cases concerning extra-trial disclosure, see Annot., 17 A.L.R.2d 383 (1951).

80 Charles of the Ritz Dist. Corp. v. Federal Trade Com'n, 143 F.2d 676 (2d Cir. 1944); Shimadzu v. Electric Storage Battery Co., 6 F.Supp. 393 (E.D.Pa. 1934) (subsequent citations omitted) ; Grasselli Chemical Co. v. National Aniline \& Chemical Co., 282 Fed. 379 (S.D. N.Y. 1920) ; Johnson Steel Street-Rail Co. v. North Branch Steel Co., 48 Fed. 191 (W.D.Pa. 1891) (third parties may find their trade secrets revealed in trials to which they are not even parties); 8 WIGMORE, EvIDENCE $\$ 2212$ (3rd ed. 1940).

90 DuPont Powder Co. v. Masland, 244 U.S. 100, 103 (1917).

01 The formula was required in Coca-Cola Co. v. Joseph C. Wirtbman Drug Co., 48 F.2d 743 (8th Cir. 1931) ; Putney v. DuBois Co., 240 Mo. App. 1075, 226 S.W.2d 737 (1950); Hillick v. E. W. Edwards \& Son, 143 Misc. 277, 256 N.Y. Supp. 313 (1932), modified, 257 N.Y. Supp. 945 (1932).

Only a qualitative disclosure of ingredients was required in Lenerts v. Rapidol Distributing Corporation, 3 F.R.D. 42 (N.D.N.Y. 1942) ; Spain v. United States Rubber Co., 94 N.H. 342, 54 A.2d 364 (1947). If analysis will reveal the ingredients, the court inay deny disclosure by the inventor. Drake v. Herrman, 261 N.Y. 414, 185 N.E. 685 (1933) ; Gehm v. Countess Moritza Cosmetic Co., 196 Misc. 785, 95 N.Y.S.2đ 754 (1949) ; Kaplan v. Roux Laboratories, 273 App. Div. 865,76 N.Y.S.2d 601 (1948).

02 Shimadzu v. Electric Storage Battery Co., 6 F. Supp. 393 (E.D. Pa. 1934) (subsequent citations omitted) ; Rowell v. William Koebl Co., 194 Fed. 446 (W.D. N.Y. 1912), 216 Fed. 780 (W.D.N.Y. 1914), rev'd on other grounds, 240 Fed. 953 (2d Cir. 1917) ; Bead Chain Mfg. Co. v. Smith, 1 N.J. 118, 62 A.2d 215 (1948); Griffin Mfg. Co. v. Gold Dust Corporation, 245 App. Div. 385, 282 N.Y.Supp. 931 (1935).

${ }^{93}$ American Seal-Kap Corp. v. Smith Lee Co., 162 Misc. 701, 295 N.Y.Supp. 381 (1937); Meyer Bros. Inc. v. Higgins, 232 App. Div. 783, 249 N.Y.Supp. 921 (1931).

84 N.D. Q. Specialty Corp. v. Cypress Novelty Corp., 250 App. Div. 881, 295 N.Y. Supp. 218 (1937); American Seal-Kap Corp. v. Smith Lee Co., 154 Misc. 176, 277 N.Y. Supp. 549 (1935) (subsequent citations omitted).

05 Segal Lock \& Hardware Co. v. Federal Trade Com'n, 143 F.2d 935 (2d Cir. 1944), cert. denied, 323 U.S. 791 (1945) ; John T. Lloyd Laboratories, Inc. v. Lloyd Bros. Pharm., 131 F.2d 703 (6th Cir. 1942) ; A. O. Smith Corporation v. Petroleum Iron Works Co., 73 F.2d 531 (6th
} 
The general approach to the problem of secrecy as herein described is adhered to in the Federal Rules of Civil Procedure. ${ }^{96}$

\section{Rights of the employer in the employee's invention}

The inventor who is an employee may have to contend with an employer's assertion of rights to his invention. The relationship of employer and employee per se does not prescribe any rights of the former in inventions of the latter. ${ }^{97}$ Depending upon the circumstances surrounding the act of invention, the employer's interest may consist of complete ownership, limited rights concerning practice of the invention (shop rights), or no rights whatsoever. ${ }^{98}$

Contracts of employment may expressly provide that all rights of an employee in his inventions, including the mchoate right to patents, ${ }^{99}$ shall become the property of the employer. ${ }^{100}$ Implied agreements of similar

Cir. 1934), modified, 74 F.2d 934 (6th Cir. 1935) (record was "sealed"); Herold v. Herold China \& Pottery Co., 257 Fed. 911 (6th Cir. 1919) ; Stone v. Goss, 65 N.J. Eq. 756, 55 Atl. 736 (1903). In Remington Rand v. Control Instrument Co., 7 F.R.D. 18 (E.D. N.Y. 1947) both parties filed disclosures in sealed envelopes for simultaneous opening by the court.

00 FED. R. CIV. P. 26-37. See Annot., 17 A.L.R.2d 383 (1951).

07 U.S. v. Dubilier Condenser Corp., 289 U.S. 178, 187 (1932) ("The respective rights and obligations of employer and employee, touching an invention conceived by the latter, spring from the contract of employment."); Solomons v. United States, 137 U.S. 342 (1890) ; Marsliall v. Colgate-Palmolive-Peet Co., 175 F.2d 215 (3rd Cir. 1949) ; Wireless Specialty Apparatus Co. v. Mica Condenser Co., 239 Mass. 158, 131 N.E. 307 (1921) ; Clerke v. Beck, 13 N.J. Super. 73, 80 A.2d 252, 253 (1951) ("The mere fact that an invention was conceived and developed while the inventor was employed by another does not give to the employer any right in or title to the invention."). See Snelling, The Rights of an Inventor-Employee, 22 J. Pat. OFF. Soc'y 410 (1940).

08 The development of the shop rights doctrine is well presented in a series of Annot., 16 A.L.R. 1177 (1922), 32 A.L.R. 1037 (1924), 44 A.L.R. 593 (1926), 85 A.L.R. 1512 (1933), 153 A.L.R. 983 (1944). See also 35 AMr. JuR., Master and Servant \$\$ 90-95 (1941) ; 56 C.J.S., Master and Servant $\S 73$ (1948); Notes, 28 Tex. L. Rev. 728 (1950), 30 ColvM. L. Rev. 1172 (1930), 12 MrNn. L. Rev. 670 (1928); Restatement, Agency $\$ 397$ (1933).

09 Dalzell v. Dueber Manufacturing Co., 149 U.S. 315 (1892); Cammeyer v. Newton, 94 U.S. (4 Otto) 225 (1876) ; Railroad Company v. Trimble, 77 U.S. (10 Wall.) 367 (1870); Universal Winding Co. v. Clarke, 108 F.Supp. 329 (D. Conn. 1952) ; New Era Electric Range Co. v. Serrell, 252 N.Y. 107, 169 N.E. 105 (1929) ; Burr v. De La Vergne, 102 N.Y. 415, 7 N.E. 366 (1886).

The court may require the employee to apply for the patent in the absence of good reason why he should not. Guth v. Minnesota Mining \& Mfg. Co., 72 F.2d 385 (7th Cir. 1934), cert. denied, 294 U.S. 711 (1935).

100 Dalzell v. Dueber Manufacturing Co., 149 U.S. 315 (1892) ; Hebbard v. American Zinc, Lead \& Sinelting Co., 161 F.2d 339 (8th Cir. 1947) ; Paley v. DuPont Rayon Co., 71 F.2d 856 (7th Cir. 1934); New Jersey Zinc Co. v. Singmaster, 71 F.2d 277 (2d Cir. 1934), cert. denied, 293 U.S. 591 (1934); Conway v. White, 292 Fed. 837 (2d Cir. 1923), 300 Fed. 866 (D. Conn. 1924), rev'd on other grounds, 9 F.2d 863 (2d Cir. 1925); Mississippi Glass Co. v. Franzen, 143 Fed. 501 (3rd Cir. 1906); Thibodeau v. Hildreth, 124 Fed. 892 (1st Cir. 1903).

A contract requiring the assignment of rights to an invention made during employment does not include inventions made theretofore. Rotary Lift Company v. Clayton, 127 F. Supp. 176 (D. Mass. 1954) [noted, 23 Geo. Wase. L. REv. 488 (1955)] ; Fox v. Kingsland, 81 F. Supp. 
nature may be found from the nature of the employment, ${ }^{101}$ especially where a primary part of the employee's duty has been research development and invention. ${ }^{102}$ The validity of such contracts is conceded and enforcement will be by specific performance ${ }^{103}$ although the exact interpretation and scope of particular contracts may first require judicial determination. ${ }^{104}$ The employee can be compelled to disclose his secret discov-

433 (D.D.C. 1948), aff'd, 180 F.2d 45 (D.C.C. 1950); Quaker State Oil Refining Co. v. Talbot, 315 Pa. 517, 174 Atl. 99 (1934), 322 Pa. 155, 185 Atl. 586 (1936) ; Sharples v. McCornack, 254 Pa. 535, 99 Atl. 153 (1916) ; cf. Conway v. White, 9 F.2d 863 (2d Cir. 1925), reversing, 300 Fed. 866 (D. Conn. 1924).

Post-employment inventions can be specifically included if the contract is fair [Gas Tool Patents Corporation v. Mould, 133 F.2d 815 (7th Cir. 1943); Hulse v. Bonsack Mach. Co., 65 Fed. 864 (4th Cir. 1895); but cf. Guth v. Minnesota Mining \& Mfg. Co., 72 F.2d 385 (7th Cir. 1934), cert. denied, 294 U.S. 711 (1935)] as can patents issued after termination of the employment [Bolin v. Wilkes, 249 Fed. 696 (5th Cir. 1918)].

See Knoth, Assignment of Future Inventions, 27 CHI-KENT L. REv. 295 (1949); Knoth, Assignments of Future Inventions, Particularly as Between Employers and Employees, $31 \mathrm{~J}$. Pat. OfF. SoC'y 532 (1949); Note, N.Y.U. Intra. L. Rev. 58 (1949).

101 Standard Parts Co. v. Peck, 264 U.S. 52 (1923); Marshall v. Colgate-Palmolive-Pect Co., 175 F.2d 215 (3rd Cir. 1949) ; Dinwiddie v. St. Louis \& O'Fallon Coal Co., 64 F.2d 303 (4th Cir. 1933); Magnetic Mfg. Co. v. Dings Magnetic Separator Co., 16 F.2d 739 (7th Cir. 1926), cert. denied, 274 U.S. 740 (1927); State v. Neal, 152 Fla. 582, 12 So.2d 590 (1943), cert. denied, 320 U.S. 783 (1943), 154 Fla. 326, 17 So.2d 703 (1944); Pomeroy Ink Co. v. Pomeroy, .77 N.J. Eq. 293, 78 Atl. 698 (1910). See Schlaffman, Employer's Rights in the Patentable Inventions of His Employees, 1 ST. Lours U.L.J. 1 (1949); Note, 22 Notre DaMe LAW. 429 (1947).

102 U.S. v. Dubilier Condenser Corp., 289 U.S. 178, 187 (1932) ("One employed to inake an invention, who succeeds, during his term of service, in accomplisbing that task, is bound to assign to his employer any patent obtained."); Solomons v. Umited States, 137 U.S. 342 (1890); Houghton v. United States, 23 F.2d 386 (4th Cir. 1928), cert. denied, 277 U.S. 592 (1928); North American Philips Co. v. Brownshield, 111 F. Supp. 762 (S.D.N.X. 1953); Crown Cork \& Seal Co. v. Fankhanel, 49 F. Supp. 611 (D. Md. 1943) ; National Development Co. v. Gray, 316 Mass. 240, 55 N.E.2d 783 (1944); Marcalus Mfg. Co. v. Sulkvan, 142 N.J. Eq. 434, 60 A.2d 330 (1948); cf. Gill v. United States, 160 U.S. 426 (1896).

${ }_{103}$ Standard Parts Co. v. Peck, 264 U.S. 52, 59 (1923) ("It cannot be contended that the invention of a specific thing cannot be made the subject of a bargain and pass in execution of it.") ; Railroad Company v. Trimble, 77 U.S. (10 Wall.) 367 (1870); Guth v. Minnesota Mining \& Mfg. Co., 72 F.2d 385 (7th Cir. 1934), cert. denied, 294 U.S. 711 (1935); Paley v. DuPont Rayon Co., 71 F.2d 856 (7th Cir. 1934); New Jersey Zinc Co. v. Singmaster, 71 F.2d 277 (2d Cir. 1934), cert. denied, 293 U.S. 591 (1934); Conway v. White, 292 Fed. 837 (2d Cir. 1923), 300 Fed. 866 (D. Conn. 1924), rev'd on other grounds, 9 F.2d 863 (2d Cir. 1925); Wege v. Safe-Cabinet Co., 249 Fed. 696 (6th Cir. 1918); Mississippi Glass Co. v. Franzen, 143 Fed. 501 (3rd Cir. 1906); Velsicol Corporation v. Hyman, 338 Ill. App. 52, 87 N.E.2d 35 (1949), rev'd on other grounds, 405 Ill. 352, 90 N.E.2d 717 (1950), cert. denied, 339 U.S. 966 (1950); Valley Iron Works Mfg. Co. v. Goodrick, 103 Wisc. 436, 78 N.W. 1096 (1899). Sce Note, 40 HARV. L. REV. 1000 (1927).

104 Mississippi Glass Co. v. Franzen, 143 Fed. 501 (3rd Cir. 1906); Universal Winding Co. v. Clarke, 108 F. Supp. 329 (D. Conn. 1952); Hirshhorn v. Mine Safety Appliances Co., 106 F. Supp. 594 (W.D.Pa. 1952) (subsequent citations omitted); National Development Co. v. Gray, 316 Mass. 240, 55 N.E.2d 783 (1944); White Heat Products Co. v. Thomas, 266 Pa. 551, 109 Atl. 685 (1920). 
eries to his employer alone. ${ }^{105}$ An employer's indifference to a proffered invention may lead to a forfeiture of his rights therein. ${ }^{106}$

In the absence of an express or implied agreement, the courts will not give the employer sole rights to the employee's invention; ${ }^{107}$ the circumstances of the employment are often deemed insufficient to justify such a deprivation of all the employee's rights to the invention. ${ }^{108}$ In such cases the extent of the "shop right" accorded an employer is determined by state ${ }^{1 a w^{109}}$ and depends upon the relationship between the employment and the invention. ${ }^{110}$ Shop rights may arise where the employee made the invention during his hours of employment, where he used the employer's materials, where he received assistance from co-employees or where he acquiesced in the practice of the invention by the employer. ${ }^{111}$ These limited rights normally include the royalty-free, non-exclusive and non-assignable use of the invention; ${ }^{112}$ in appropriate cases the privilege may also be given

105 Thibodeau v. Hildreth, 124 Fed. 892 (1st Cir. 1903); Westervelt v. National Paper \& Supply Co., 154 Ind. 673, 57 N.E. 552 (1900); Wireless Specialty Apparatus Co. v. Mica Condenser Co., 239 Mass. 158, 131 N.E. 307 (1921) ; Silver Spring Bleaching \& Dyeing Co. v. Woolworth, 16 R.I. 729, 19 Atl. 528 (1890).

106 Parker Rust-Proof Co. v. Allen, 231 Mich. 69, 203 N.W. 890 (1924) ; cf. Paley v. DuPont Rayon Co., 71 F.2d 856 (7th Cir. 1934), affirming, 4 F. Supp. 290 (N.D. Ml. 1933).

107 U.S. v. Dubilier Condenser Corp., 289 U.S. 178, 187 (1932) ("II]f the employment be general, albeit it cover a field of labor and effort in the performance of which the employee conceived the invention for which he obtained a patent, the contract is not so broadly construed as to require an assignment of the patent."); Dalzell v. Dueber Manufacturing Co., 149 U.S. 315 (1892) ; Hapgood v. Hewitt, 119 U.S. 226 (1886) ; Pressed Steel Car Co. v. Hansen, 137 Fed. 403 (3rd Cir. 1905), cert. denied, 199 U.S. 608 (1905); Manton-Gaulin Mfg. Co. v. Colony, 255 Mass. 194, 151 N.E. 71 (1926). See Note, 1 Geo. WASH. L. REv. 148 (1932).

108 Solomons v. United States, 137 U.S. 342 (1890); Hapgood v. Hewitt, 119 U.S. 226 (1886); Ingle v. Landis Tool Co., 272 Fed. 464 (3rd Cir. 1921), cert. denied, 257 U.S. 644 (1921); Barlow \& Seelig Mfg. Co. v. Patch, 232 Wisc. 220, 286 N.W. 577 (1939), 236 Wisc. 223, 295 N.W. 39 (1940).

109 Hirshhorn v. Mine Safety Appliances Co., 106 F. Supp. 594 (W.D.Pa. 1952) (subsequent citations omitted) ; Toner v. Sobelman, 86 F. Supp. 369 (E.D.Pa. 1949), 97 F. Supp. 503 (E.D.Pa. 1951).

110 See note 114 infra. See Note, 22 MnNo. L. Rev. 115 (1937).

111 U.S. v. Dubilier Condenser Corp., 289 U.S. 178, 188 (1932) ("[W] here a servant, during his hours of einployment, working with his master's inaterials and appliances, conceives and perfects an invention for which he obtains a patent, he must accord his master a non-exclusive right to practice the invention."); Gill v. United States, 160 U.S. 426 (1896); Lane \& Bodley Co. v. Locke, 150 U.S. 193 (1893); Solonions v. United States, 137 U.S. 342 (1890); McClurg et al. v. Kingsland et al., 42 U.S. (1 How.) 202 (1843); Grip Nut Co. v. Sharp, 150 F.2d 192 (7th Cir. 1945), cert. denied, 326 U.S. 742 (1945); Blauvelt v. Interior Conduit \& Insulation Co., 80 Fed. 906 ( $2 \mathrm{~d} \mathrm{Cir.} \mathrm{1897).} \mathrm{The} \mathrm{enployer} \mathrm{may} \mathrm{also} \mathrm{have} \mathrm{a} \mathrm{breach} \mathrm{of} \mathrm{contract} \mathrm{action} \mathrm{against}$ an employee for time spent in unauthorized "inventing." American Stay Co. v. Delaney, 211 Mass. 229, 97 N.E. 911 (1912).

112 U.S. v. Dubilier Condenser Corp., 289 U.S. 178 (1932) (non-exclusive); Gill v. United States, 160 U.S. 426 (1896) (royalty-free); Solomons v. United States, 137 U.S. 342 (1890); Hapgood v. Hewitt, 119 U.S. 226 (1886) (non-assignable); Pressed Steel Car Co. v. Hansen, 137 Fed. 403 (3rd Cir. 1905), cert. denied, 199 U.S. 608 (1905); Toner v. Sobelman, 86 F. Supp. 369 (E.D. Pa. 1949), 97 F. Supp. 503 (E.D. Pa. 1951). 
of making and selling the invention. ${ }^{113}$ No precise correlation exists between the circumstances of the employment and the scope of the rights to be granted an employer ; ${ }^{114}$ in general, the employer's rights can be expected to be made coextensive with the needs of his business. ${ }^{115}$

It is also possible that the employer may acquire no rights whatsoever in his employee's invention. This would be the expected result, for instance, where the employee has created the invention on his own time at his own expense and has continuously asserted his personal rights to the invention. ${ }^{110}$

113 Hapgood v. Hewitt, 119 U.S. 226 (1886); Consolidated Vultee A. Corp. v. Maurice A. Garbell, Inc., 204 F.2d 946 (9th Cir. 1953), cert. denied, 346 U.S. 873 (1953); Flannery Bolt Co. v. Flannery, 86 F.2d 43 (3rd Cir. 1936), 108 F.2d 531 (3rd Cir. 1939), cert. denied, 309 U.S. 671 (1940); Withington-Cooley Manuf'g Co. v. Kinney, 68 Fed. 500 (6th Cir. 1895); Valley Iron Works Mfg. Co. v. Goodrick, 103 Wisc. 436, 78 N.W. 1096 (1899); Fuller \& Jolnson Manuf'g Co. v. Bartlett, 68 Wisc. 73, 31 N.W. 747 (1887).

114 Flannery Bolt Co. v. Flannery, 86 F.2d 43, 44 (3rd Cir. 1936) ("The scope of a shopright must be determined from the nature of the employer's business, the character of the invention involved, the circuunstances which created it and the relation, conduct, and intention of the parties."); Barber v. National Carbon Co., 129 Fed. 370 (6th Cir. 1904) ; Toner v. Sobelman, 86 F. Supp. 369 (E.D.Pa. 1949), 97 F. Supp. 503 (E.D. Pa. 1951).

115 U.S. v. Dubilier Condenser Corp., 289 U.S. 178, 189 (1932) ("[T]lle [employer] is in equity entitled to use [the invention] and to duplicate it as often as lie may find occasion to employ sinilar appliances in lis business."); Grip Nut Co. v. Sharp, 150 F.2d 192 (7th Cir. 1945), cert. denied, 326 U.S. 742 (1945); Wiegand v. Dover Mfg. Co., 292 Fed. 258 (N.D. Ohio 1923).

116 Matarese v. Moore-McCormack Limes, 158 F.2d 631 (2d Cir. 1946) ; Sinall v. HeywoodWakefield Co., 13 F.Supp. 825 (D. Mass. 1936) (subsequent citations omitted); Burton v. Burton Stock-Car Co., 171 Mass. 437, 50 N.E. 1029 (1898); Clerke v. Beck, 13 N.J. Super. 73, 80 A.2d 252 (1951) ; Doscher v. Phelps Guardant Time Lock Co., 89 Misc. 561, 153 N.Y. Supp. 710 (1915), aff'd, 224 N.Y. 718, 121 N.E. 865 (1918); McNamara v. Powell, 256 App. Div. 554, 11 N.Y.S.2d 491 (1939); Gemco Engincering \& Mfg. Co. v. Henderson, 151 Ohio St. 95, 84 N.E.2d 596 (1949). The employment contract can be drafted to include inventions made at home. Toledo Machine \& Tool Co. v. Byerlein, 9 F.2d 279 (6th Cir. 1925); Detroit Lubricator Co. v. Lavigne Mfg. Co., 151 Micl. 650, 115 N.W. 988 (1908). 